\begin{tabular}{|c|c|c|c|c|c|c|c|c|c|}
\hline \multirow{2}{*}{1910} & \multicolumn{9}{|c|}{ Perihelzcit } \\
\hline & Sept. 12 & Sept. 20 & Sept. 28 & Okt. 6 & Okt. I4 & Okt. 22 & Okt. 30 & Nov. 7 & Nov. 15 \\
\hline Nov. 31 & $\begin{array}{c}7^{\mathrm{h}} 3^{6^{\mathrm{m}}} \cdot 4 \\
+36^{\circ} 18^{\prime} \\
0.87\end{array}$ & $\begin{array}{c}7^{\mathrm{h}} \mathrm{I}^{\mathrm{m}} \cdot 7 \\
+36^{\circ} 45^{\prime} \\
\quad 0.95\end{array}$ & $\begin{array}{r}6^{\mathrm{h}} 5 \mathrm{I}^{\mathrm{m}} \cdot 2 \\
+36^{\circ} 57^{\prime} \\
\\
1.04\end{array}$ & $\begin{array}{l}6^{\mathrm{h}} 26^{\mathrm{m}} \cdot 2 \\
+36^{\circ} 5^{2} \\
\quad \text { I. } 12\end{array}$ & $\begin{array}{c}6^{\mathrm{h}} \mathrm{o}^{\mathrm{m}} \mathrm{I} \\
+36^{\circ} 27^{\prime} \\
\mathrm{I} .2 \mathrm{I}\end{array}$ & $\begin{aligned} & 5^{h} 33^{m} \cdot 2 \\
&+ 35^{\circ} 38^{\prime} \\
& 1.28\end{aligned}$ & $\begin{array}{c}5^{\mathrm{h}} 5^{\mathrm{m}} \cdot 8 \\
+34^{\circ} 25^{\prime} \\
\\
\\
1.34\end{array}$ & $\begin{aligned} & 4^{\mathrm{h}} 3^{8^{\mathrm{m}}} \cdot 5 \\
&+ 32^{\circ} 49^{\prime} \\
& \mathrm{I} \cdot 39\end{aligned}$ & $\begin{array}{c}4^{\mathrm{h}} \mathrm{II}^{\mathrm{m}} \cdot 8 \\
+30^{\circ} 49^{\prime} \\
\text { I. } 4 \mathrm{I}\end{array}$ \\
\hline
\end{tabular}

Triest, r 9 I० Juni 2 I.

F. Hopfner.

\title{
Elementi ellittici ed effemeride di (654) Zelinda in terza opposizione.
}

Lasciando invariati gli elementi ellittici che ebbi con tre osservazioni di Roma (C. R.) in prima opposizione (le differenze fra calcolo ed osservazione essendo in seconda opposizione minime, messe in conto le perturbazioni per Giove e Saturno) ho osculato per la terza opposizione alla data I 9 ro Nov. 8 prossima a quella, includendo le perturbazioni sempre per Giove e Saturno.

L'astro potrà essere osservato con facilità per mezzo della seguente effemeride.
Elementi in terza opposizione.

Epoca I9 1o Novembre $8 \times 2^{\text {h }}$ Berlino.

$$
\begin{aligned}
& M=280^{\circ} 12^{\prime} \quad 5^{\prime \prime} \cdot 9 \\
& \omega=2 \mathrm{I} 22 \mathrm{I} 13.6 \\
& \left.\delta_{0}=278.1531 .2\right\} \text { I910.0 } \\
& i=\text { I8 } 19028.9 \\
& \varphi=131534.2 \\
& \mu=\text { IoI9".2974 } \\
& \log a=0.361137
\end{aligned}
$$

Effemeride a r $^{\mathrm{h}}$ Berlino

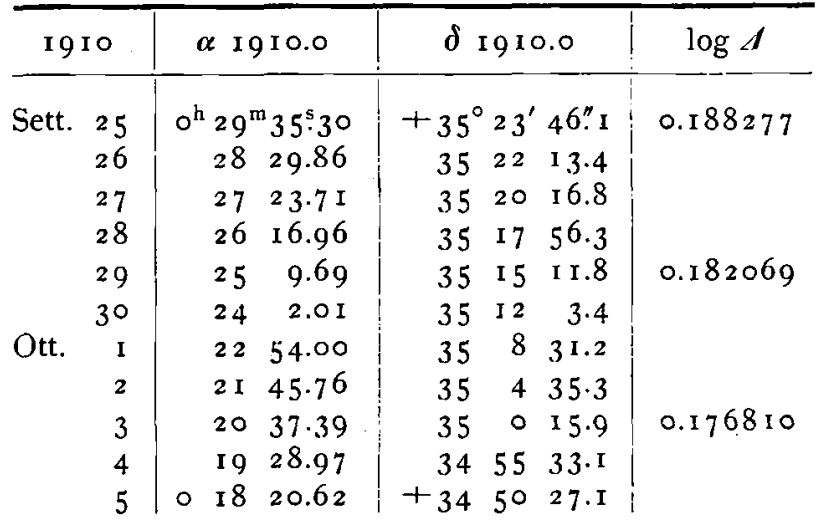

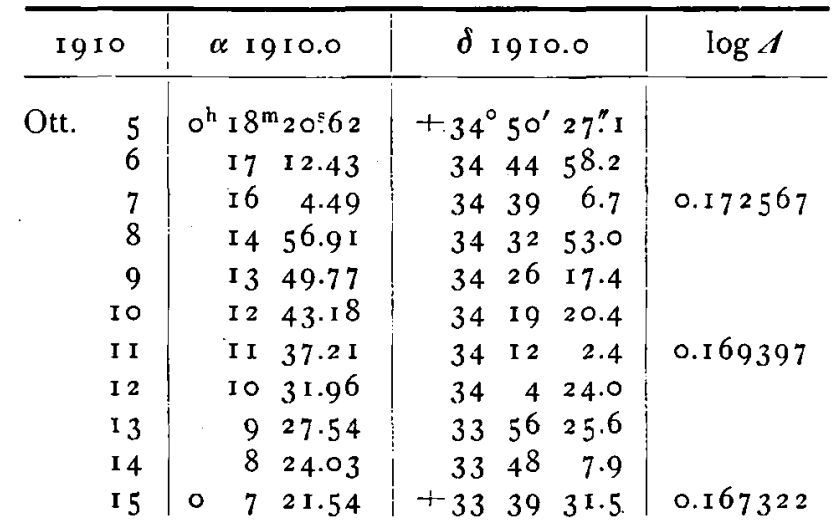

Opposizione in $\alpha$ alla data 30 Settembre. Grandezza $={ }_{1}{ }^{\mathrm{m}} \cdot 5$.

Per il calcolo dell'effemeride, all'infuori dei limiti di tempo soprascritti, valgono i costanti ausiliari seguenti:

$$
\begin{aligned}
& x=[9.978298] \cdot r \cdot \operatorname{sen}\left(v+221^{\circ} 2^{\prime} 19^{\prime \prime} \cdot 7\right) \\
& \left.y=\left[9.95803^{2}\right] \cdot r \cdot \operatorname{sen}(v+1222544.8)\right\} \text { I } 9 \text { 1 } 0.0 \\
& z=[9.716368] \cdot r \cdot \operatorname{sen}(v+163 \text { I0 } 32.6)\}
\end{aligned}
$$

Rocca di Papa, I9 пo Giulio 24.

\section{E. Millosevich.}

\begin{tabular}{|c|c|c|c|}
\hline 1910 & & $\alpha$ & \\
\hline Sept. I & $17^{\mathrm{b}}$ & & $29^{5}$ \\
\hline 3 & I 7 & Io & 13 \\
\hline 5 & 17 & I 6 & 10 \\
\hline 7 & I 7 & 22 & 22 \\
\hline 9 & I 7 & 28 & 47 \\
\hline I I & 17 & 35 & 26 \\
\hline I 3 & 17 & 42 & I 8 \\
\hline I 5 & 17 & 49 & 24 \\
\hline
\end{tabular}

\section{Auffindung des d'Arrestschen Kometen $1910 \mathrm{c}$.}

Am 27. August erhielt die Zentralstelle folgendes Telegramm aus Algier:

"Comète d'Arrest 26 Août $9^{\mathrm{h}} 3^{\mathrm{m}} \cdot 6 \mathrm{t}$ t. m. Alger $\alpha$ app. ${ }_{1} 6^{\mathrm{h}} 48^{\mathrm{m}} 25^{\mathrm{s}} \cdot 33 \delta$ app. $-9^{\circ} 42^{\prime} 5^{\prime \prime}$. Grand. I $4^{\mathrm{m}}$. Gonnessiat. "

Die Korrektion der Ephemeride von G. Leveau im Bull. Astr. 27.8 $\mathrm{I}$ beträgt hiernach $-\mathrm{I}^{\mathrm{m}} \mathrm{I} 7^{\mathrm{s}}+5 \cdot \mathrm{I}$. Für die nächste Zeit gibt diese Ephemeride für $0^{\text {h }}$ M. Z. Paris folgende Örter:

$\begin{array}{cc}\log \Delta & 1: r^{2} \Delta^{2} \\ 9.8227 & 1.37 \\ 9.8236 & \\ 9.8247 & 1.38 \\ 9.8260 & \\ 9.8276 & 1.37 \\ 9.8294 & \\ 9.83 \times 5 & 1.35 \\ 9.8338 & \end{array}$

In halt zu Nr. 4437. 7. Bauschinger. Vorausberechnung der Erscheinung I9I0-I I des periodischen Kometen Brooks (1889 V, I896 VI, 1903 V). 329. - F. Hopfner. Elemente und Aufsuchungsephemeride für den Kometen 1890 VII (Spitaler). 337. - E. Millosevich. Elementi ellittici ed effemeride di (654) Zelinda in terza opposizione. 343. - Auffindung des d'Arrestschen Kometen 1910 c. 343 .

Geschlossen xgro Aug. 29. Herausgeber: H. Kobold. Druck von C. Schaidt (Georg Oheim, Alfred Oheim!. Expedition: Kiel, Moltkestr. 80. 